\title{
OPTIMIZED MAPPING AND NAVIGATION OF REMOTE AREA THROUGH AN AUTONOMOUS ROBOT
}

\author{
P. P. Chavan ${ }^{1}$, R.D. Kulkarni ${ }^{2}$, G. S. Nathani ${ }^{3}$, S. S. Desai ${ }^{4}$ \\ ${ }^{1}$ Assistant Prof, Department of Electronics \& Telecommunication, $K J$ College of Engineering \& Management Research, \\ Pune, Maharashtra, India \\ ${ }^{2}$ Student of Department of Electronics \& Telecommunication, KJ College of Engineering \& Management Research, \\ Pune, Maharashtra, India \\ ${ }^{3}$ Student of Department of Electronics \& Telecommunication, KJ College of Engineering \& Management Research, \\ Pune, Maharashtra, India \\ ${ }^{4}$ Student of Department of Electronics \& Telecommunication, KJ College of Engineering \& Management Research, \\ Pune, Maharashtra, India
}

\begin{abstract}
Navigation and mapping are the two integrated system for an intelligent robotic unit. The "Optimized mapping and navigation of remote area through an autonomous robot" comprises of two parts, the primary unit is a four wheeled robot which is a fully automated system and the secondary unit is a user interface system developed on the JAVA platform. The developments of these units are made keeping in mind the robust requirements in different field. The robot is a fully autonomous unit, i.e. it does not require any assistance from the environment or human interaction. Proximity sensors calibrated onto the robot are used for obstacle detection process in its path. A cross reference process using mobile phone is also integrated in this project.

The robot is self sustained with algorithm to avoid any object which would lie in its path while manoeuvring across the arena. The major gist of the project is that the robot develops its own virtual grid onto the arena and hence position and align itself to it. The complete robotic unit works on this virtual grid removing necessity for any external elements to be used as a reference or support for its navigation or positioning. The secondary unit, the Graphical User Interface, developed covers all possible grounds for interaction with the user. The GUI also supports the facility of storing data onto the database for future reference or resource storing. The communication link between the two is developed by an ISP band using zigbee communication; the units are more facilitated using real time based data transfer between the two in a secure private network. This project would also demonstrate an innovative idea by using android mobile for localization and positioning of the robot.
\end{abstract}

Keywords: Robotics, Mapping \& Navigation, Wireless Communication - ZigBee, Object detection - ultrasonic proximity sensor, Android application, MIT app inventor.

\section{INTRODUCTION}

There are many strategies developed for the solution to the problem to the design of mapping and navigation of an arena ${ }^{[1]}$ ${ }^{[2]}$, the true test of these system is their capability of handling responses given to them i.e. capability of developing an robust yet powerful structure response. In many areas where a sense of navigation is required but use of external reference point or any such device that requires a setup in the targeted field cannot be accomplished is a very tedious task, and sometimes very hazardous.

With introduction of this system the robotic unit, or any similar device could set up a complete model for navigation on any ground without the need of external references ${ }^{[3]}$, this not only means an economical solution but also an overall faster resolution of the problem. Every 2-D navigation system could only be satisfied in terms of nodes or X and Y coordinates "Grid Mapping Techniques" ${ }^{[2]}$. Exception in this project is that the design is virtually calculated and these grids and nodes are all "imaginary" but they possess real time values with the reference to the initial position of the robot.

Movement of the robot is another concept, use of steady base gives it an edge to reduce error while manoeuvre across the arena. Addition to it the complete electronic design is aimed to handle any similar unit, even if the dimension or make of these hardware are changed this concept stands its ground in all scenario.

Another care is taken that the user is kept to the minimum required intervention. Reasons of isolation may be an 
advantage in area of hazards or reducing human error or designing with bare minimum complexities at the user end.

A prototype design of using an android app is also incorporated to the cause, this is explained in the impending section.

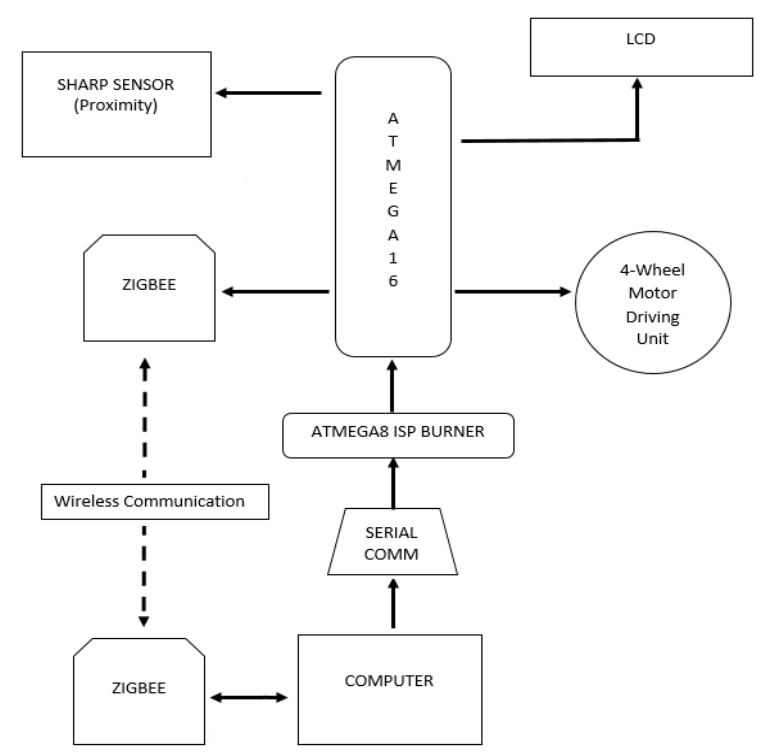

Fig 1: Basic block description of the system

\section{VIRTUAL GRID TECHNIQUE}

\subsection{Grid Mapping}

As stated above the nodes are divided among $\mathrm{X}$ and $\mathrm{Y}$ coordinates, Initially the starting node of design starts with Xnode 1 and Ynode 1, this node is the reference point to which the other nodes are to be virtually aligned accordingly. The design parameter is such that these nodes length would vary according to the user defined values.

Being independent from the use of sensor the mapping dimensions are automatically picked up as per the capability of the motor driving units of the robot, this adds to the advantage for getting an generalized throughput at the user end, as the dimension of the robot increases so is the capacity of the robotic unit.

After every movement of the robot the system updates its database with the nodes it has covered, the current node status of the design gives the idea of its active position on the grid. The graph is broadly divided into segments each on $\mathrm{Y}$ axis and $\mathrm{X}$ axis.

\subsection{Orientation Bit}

The second challenge of grid technique is to maintain the sense of orientation. The orientation changes only when the direction changes that is when the robot takes a turn in its path. The graphical display of orientation is bit as shown in fig below. The bit value of this needs to be updated after every move because this would only sense the direction which the robot faces with respect to the orientation.

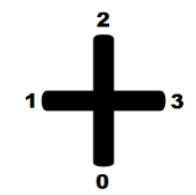

Fig 2: Orientation bit.

\section{COMMUNICATION}

\subsection{Introduction}

The server and client protocol or P2P protocol is established using ZigBee. It is a specification for a suite of high level communication protocols used to create personal area networks built from small, low-power digital radios. ZigBee based on an IEEE 802.15 standard, is low-powered buthas capability to transmit data over long distances, which is facilitated even more by using repeaters which creates a mesh network, i.e., a network with no centralized control ${ }^{[4]}$.

\subsection{ZigBee Module}

The class of communication to simplify the whole design used is Tran's receiver by securing Serial Communication using the UART (Universal Asynchronous Receiver Transmitter). The XBee/XBee-PRO OEM RF Modules could easily and readily be made compatible by RS232 standard voltage level using MAX232 IC or any voltage level converters.

By default the modules operate in Transparent Mode. When operating in this mode, the modules act as a serial line replacement - all UART data received through the DI pin is queued up for RF transmission. When RF data is received, the data is sent out the DO pin.

\subsection{Data Transmission}

Data enters the module UART through the DI pin (pin 3) as an asynchronous serial signal. The signal should idle high when no data is being transmitted. Each data byte consists of a start bit (low), 8 data bits (least significant bit first) and a stop bit (high). The figure illustrates the serial bit pattern of data passing through the module.

\subsection{Data Buffers}

When serial data enters the RF module through the DI pin (pin 3 ), the data is stored in the DI Buffer until it can be processed.When RF data is received, the data enters the DO buffer and is sent out the serial port to a host device. Once the 
DO Buffer reaches capacity, any additional incoming RF data is lost.

The CTS and RTS - Hardware Flow Control registers monitor the overflowing data buffers and respond automatically to the sender or receiver about the backward flow control initiation.

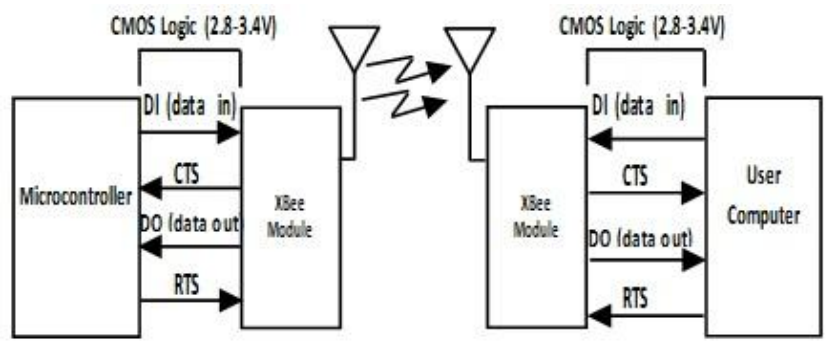

Fig3: Block Description of ZigBee module communication link with user computer.

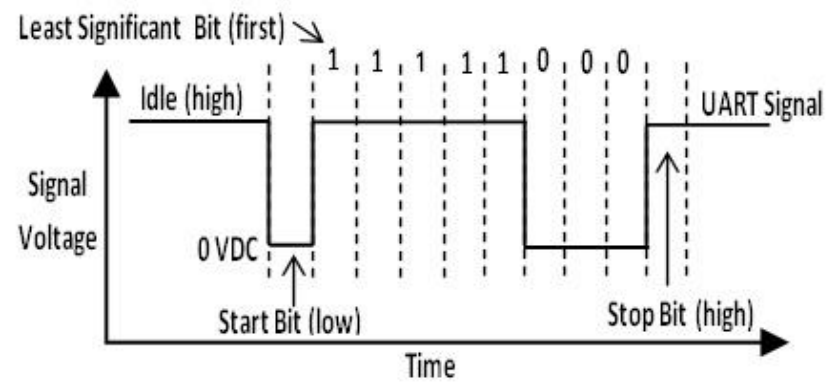

Fig 4: UART data packet as transmitted through the RF module. Example Data Format is 8 bits, No parity bit and 1 stop bit.

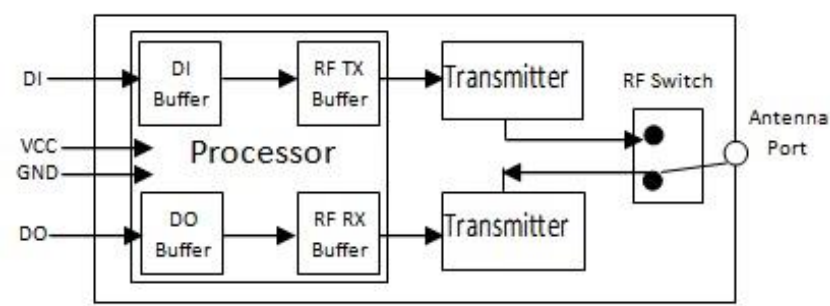

Fig 5: Internal data flow diagram - ZigBee module.

\section{THE USER INTERFERENCE}

- $\quad$ Simple GUI for user interface.

- Real time Mapping display window.

- Database available for storing resources.

- Direct serial port entities available for interaction.

- Multiple options are provided on GUI for user to control the autonomous bot.

- $\quad$ Baud rate could be easily changed and applied onto.

- No Human interaction is required once the mapping has started.
- Wide display window is available for displaying the large mapping area (Application is Robust).

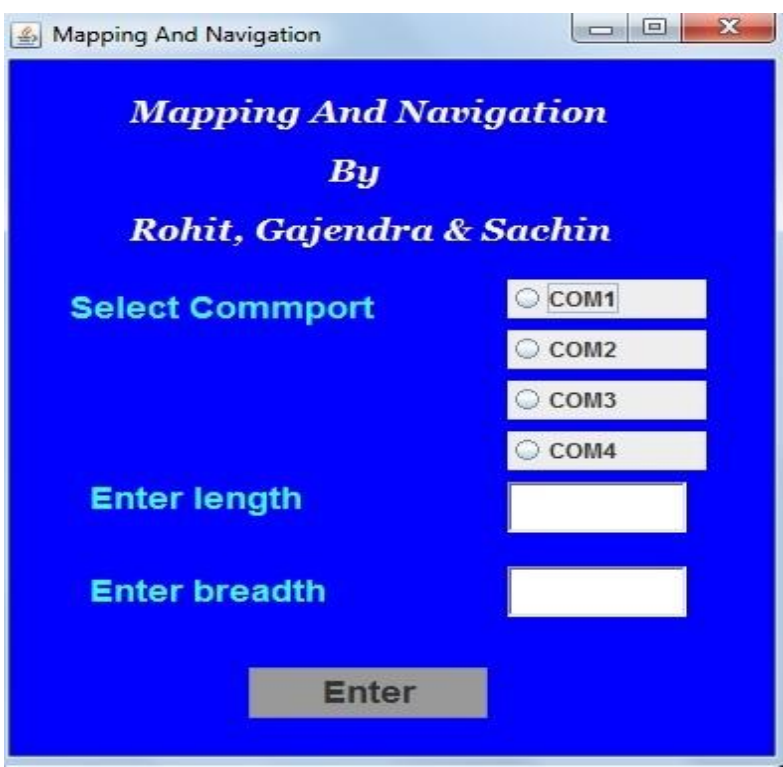

Fig 6: Graphical User Interface (GUI).

\subsection{Application Compatibility}

Application is platform independent, i.e. it can be used on any OS. The JVM (Java Virtual Machine) makes it possible with simple yet fastdesign the application does not require much of the computer resources.

\subsection{Application Requirement}

JDK package is needed in OS to create java runtime environment (JRE) ${ }^{[9]}$.

\section{MICROCONTROLLER ATMEGA32L [6]}

- High-performance, Low-power Atmel®AVR® 8-bit Microcontroller

- Advanced RISC Architecture with 131 Powerful Instructions and $32 \times 8$ General Purpose Working Registers

- High Endurance Non-volatile Memory segments including 32Kbytes of In-System Self-programmable Flash program memory, 1024Bytes EEPROM and 2Kbytes Internal SRAM.

- In-System Programming by On-chip Boot Program.

- Peripheral Features, A two 8-bit Timer/Counters with Separate Prescalers and Compare Modes, four PWM Channels, 8-channel, 10-bit ADC, Programmable Serial USART, etc.

- $\quad$ I/O and Packages contains 32 Programmable I/O Lines.

- $\quad$ Operating Voltages ranges from 2.7V - 5.5V.

- $\quad$ Speed Grades 0 - 8MHz.

- $\quad$ Power Consumption at $1 \mathrm{MHz}, 3 \mathrm{~V}, 25^{\circ} \mathrm{C}$ 


\subsection{Serial Communication}

The Universal Synchronous and Asynchronous serial Receiver and Transmitter (USART) is a highly flexible serial communication device. Port PD0 and PD1 are used as RXD and TXD pins for communication. The data are serially transferred into USART buffer registers and send for transmission or memory register as per the process call.

The figure below shows the frame format for transmission and reception of the data frames.

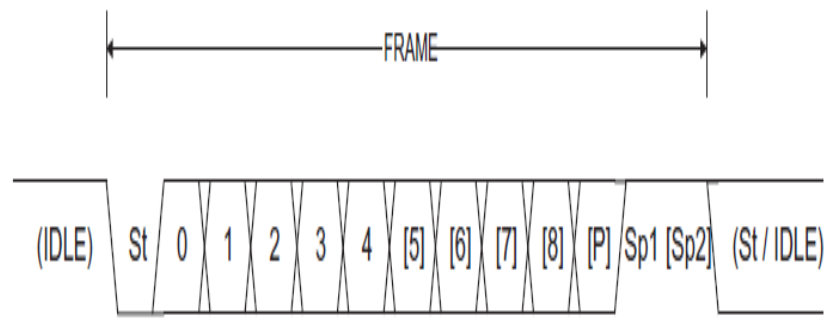

Fig 7: Frame format for USART including IDLE (idle bit), St (Start Bit), 0-8 Data bit, P (Parity bit) and SP (Stop bits).

\section{OBSTACLE DETECTION USING SENSOR}

The robot is trying to plagiarism the human senses for obstacle detection; the most economical solution to this is by introducing a proximity sensor to the robotic unit. Calculation for the parameter of distance is carried in such a way that the robot should not get lost in its path of detection and confuse its vital information of its positioning and orientation ${ }^{[5]}$.

Every possibility of detection in the complete arena is accounted into the robotic system, this design after the detection of obstacle in its path, is made by keeping track of its position on the arena, orientation, parameters of arena and to avoid the obstacle and continue to map the rest fields.

\section{WORKING}

The complete user interaction is onto the computer side of the project, the starting initiation is done using entering the field values of the system onto the GUI, as shown in Fig 9. The length and breadth of the mapping parameter. These strings are sent using xbee communication link setup to the robotic unit.

After both the units have made their first contact, the system is now ready to map the arena. Here the GUI now opens a display window onto the user screen with a start button, As soon as the start button is pressed the robotic units start sending the data resources from on ground unit to the computer section.

In case of no obstacle is detected the robotic units keeps updating its position and mark it onto the window terminal of the screen. This map building process is continuously displayed onto the screen on the real time basis ${ }^{[7]}$.

As soon as the object is detected onto the screen the robot notes the node at which it hit the object and so transfers the data back to computer end. At this point the GUI terminal unit marks the area as a red node or heavy node showing the object on the very node itself. In any case where the robot changes its motion or makes any movement the information about the system is periodically updated to the server unit on a real time basis where the mappingutility illustrates the required fields ${ }^{[8]}$, it is demonstrated in fig 8 .

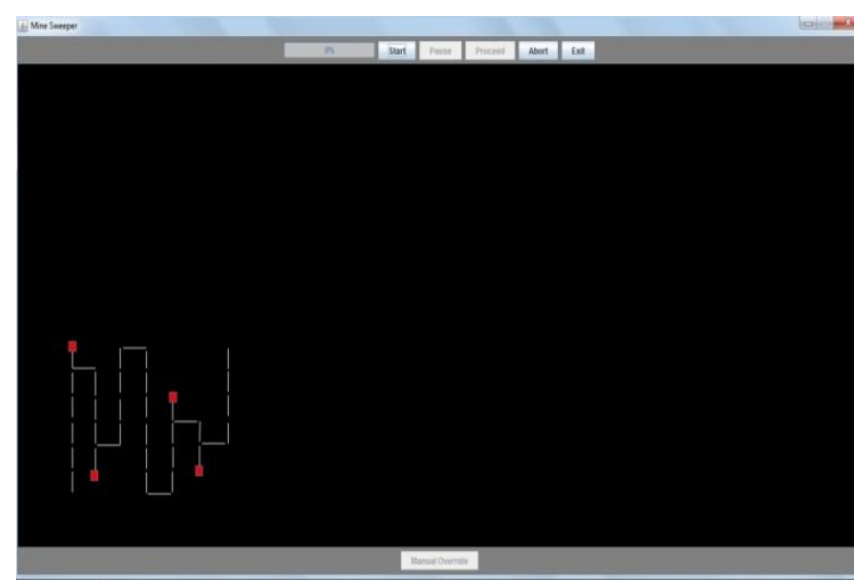

Fig 8: Map displaying terminal here the white lines denotes a clear path on those terminals and red blocks denotes an object has been detected.

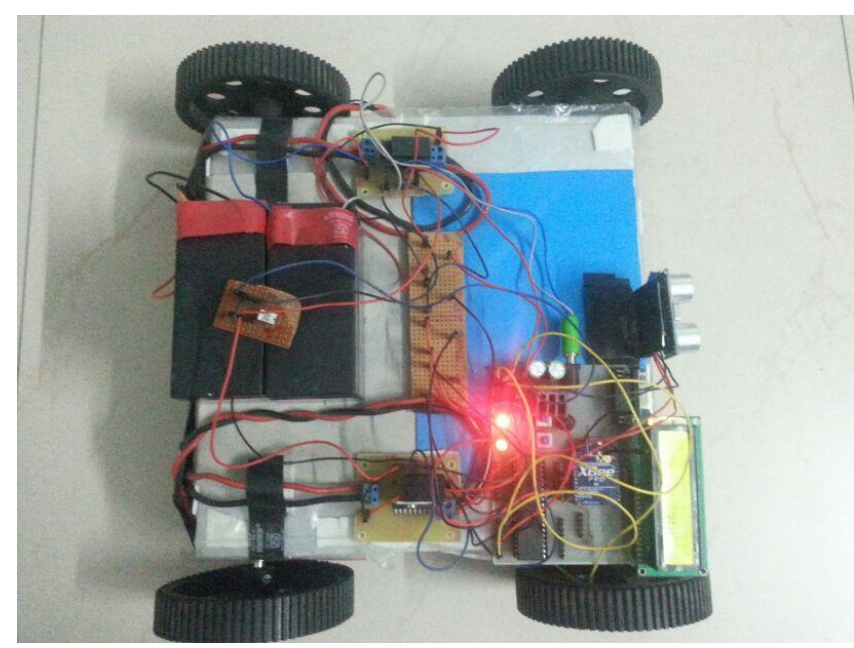

Fig 9: Robot Unit

\section{ANDROID FOR LOCALIZATION OF ROBOT}

Reliability of any system could be questioned upon as it is selfdependent and does not uses any literal means of localization from the field, to remove uncertainties and to testify the overall performance of the unit the robot could be accompanied by an 
android mobile which nowadays are available easily and at low cost.

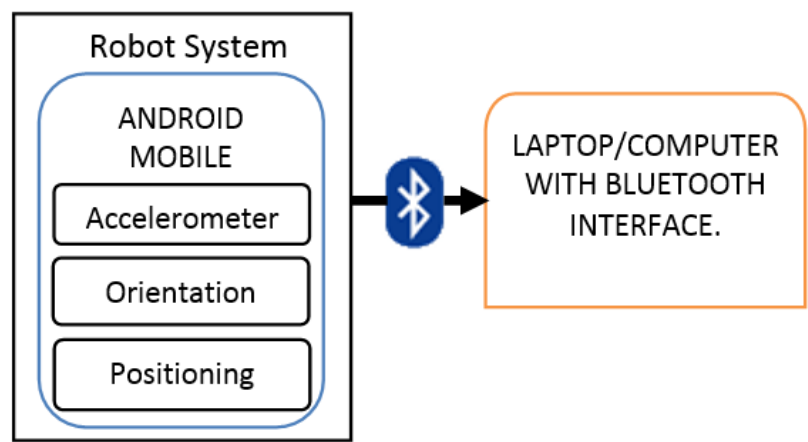

Fig 10: Block Diagram of mobile unit with server system.

\subsection{Android Sensors}

Android mobiles are loaded with sensors which are used for the operation of multiple apps onto it, the prime sensors which could be used are as following:

1. Motion sensors or Accelerometers: for measuring acceleration forces and rotational forces.

2. Orientation sensors: using magnetometers they can produce the physical position of device.

3. Location sensor: deduces the location coordinates using either GPS or network based system.

These three sensor are more than enough to vouch for the displacement and localization of robot in the unknown field.

\subsection{MIT app Inventor}

Designed under Google, and managed by MIT ${ }^{[10]}$, USA, this is an open source tool for development of android apps by users. App inventor eases the process and develops the app efficiently as well as quickly.

\subsection{Communication}

The target of designing this phase of project was to ensure reliability, the system could be more trustworthy is a different media of data communication could be used onto, the app inventor facilitates use of Bluetooth onto the device as used. The Bluetooth not only secures the transmitting data but are also available on both side of the system i.e. the mobile as well as the laptop. Hence Bluetooth was opted onto, for long ranges the device or application could be enabled with internet protocol and data could be uploaded onto the network. By default for mobile a baud rate of 9600 is used.

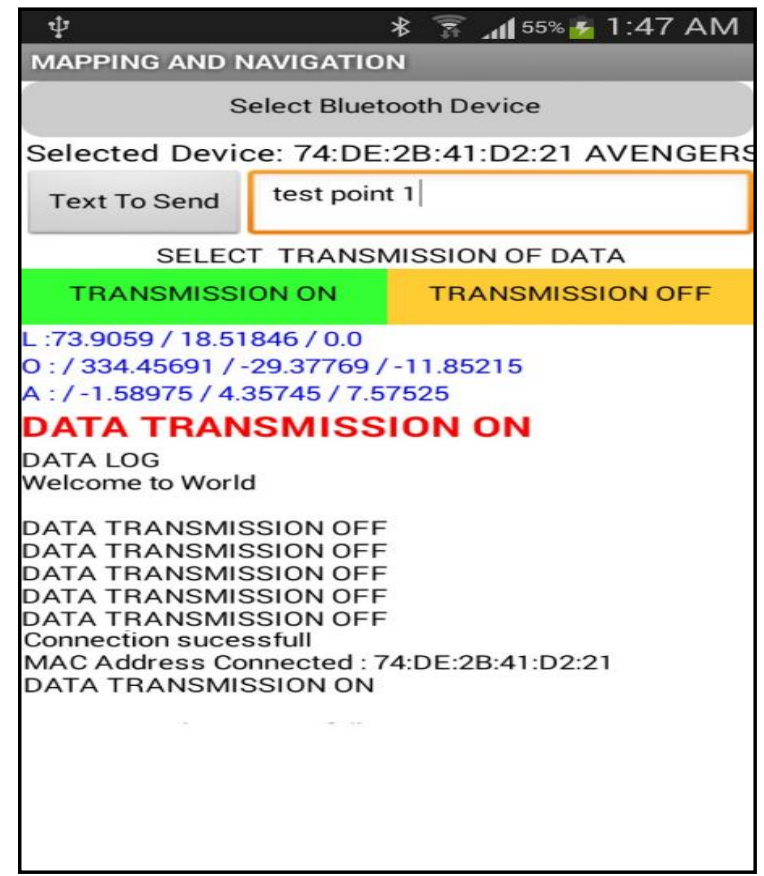

Fig 11: Screen shot of the android mobile application.

\subsection{Operation}

Data streams are send in a sequence from the android application (.apk) on the mobile placed on the robotic unit. Application after starting the Bluetooth device needs to be selected for linking, and as soon as it gets linked the unit could start sending data after the "transmission button" is clicked as shown in figure 12. The data streams arrive at terminal unit i.e. the computer system, this could be interpreted to a GUI for easy references. As shown in figure 11the terminal output of the strings. Even though application was tested on Samsung galaxy GTI9300 (S3), the application is compatible to other devices as well.

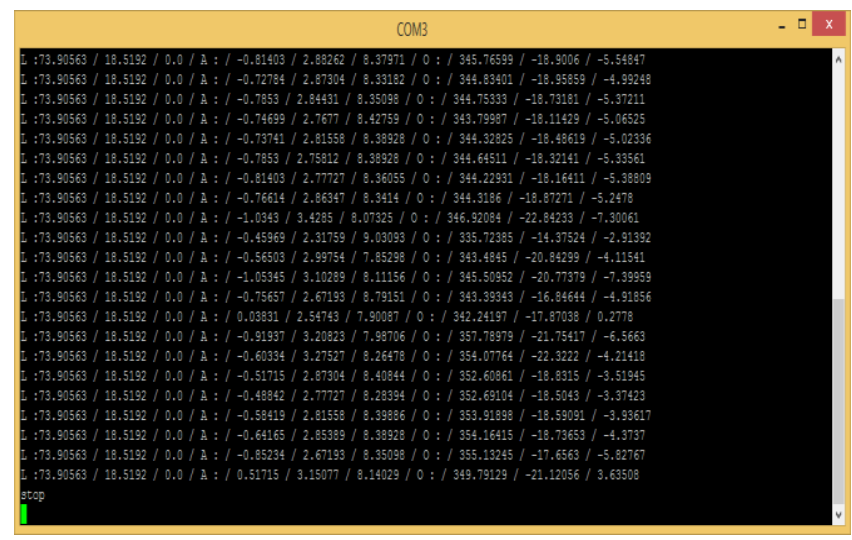

Fig 11: Server side reception of data from android unit,L strings are for location sensor followed by A strings of Accelerometer sensor and $\mathrm{O}$ string of the Orientation sensor. 


\section{APPLICATION}

- The mapping technique could be easily implemented for any robotic system.

- The robot needs no human interference during the job; this advantage could be widely explored in the areas where it's hazardous or regions where humans can't access.

- The robot is ready to map and store the complete arena which could be used as a resource database.

- Having a powerful microcontroller the system is ready to interact with multiple types of sensor and expand its application accordingly.

- The system is capable of searching objects in the complete arena autonomously, very useful and capable in search and rescue operation ${ }^{[11]}$.

- Heavy units which are transported in factories could make use of these units to calculate its path before shifting the loads.

\section{ACKNOWLEDGMENTS}

The success of any project depends upon the guidance and encouragement by the mentor and many others. I take this opportunity to express my gratitude to all those, who have been the backbone support for the successful completion of this project. This research project would not have been possible without the guidance and moral support of Prof. Mrs. P. P. Chavan, whose mentoring supervision and challenges encouraged us throughout. We appreciatively thank our HOD, Prof. Mr P. U. Chavan. His experience and expertise, and insistence, on our no less than, the best efforts, guided us to achieve our goal. We are sincerely thankful to him.

We are grateful to our institute for providing us with favourable environment and all the facilities to complete this project.We would like to dedicate this project to our family and friends who were the true pillars, both morally and monetarily. Their endless support and belief on us are the basis of our focus and dedication.

\section{REFERENCES}

[1] VikasVerma, Jaipur National University, Jagatpura, Jaipur, "Mobile robot Navigation Techniques: A Survey", Vol. 1 Issue II, September2013 ISSN: 23219653

[2] J. Borenstein ${ }^{1}$, H. R. verett ${ }^{2}$, and L. Feng ${ }^{3}$ Contributing authors: S. W. Lee and R. H. Byrne Edited and compiled by J. Borenstein - "Where am I? ,Sensors and Methods for Mobile Robot Positioning" University of Michigan For the Oak Ridge National Lab (ORNL) D\&D Program and the United States Department of Energy's Robotics Technology Development Program, April 1996

[3] JoonSeop Oh, Yoon Ho Choi, Jin Bae Park, and Yuan F. Zheng, Fellow, IEEE, "Complete Coverage
Navigation of Cleaning Robots Using Triangular-CellBased Map", IEEE TRANSACTIONS ON INDUSTRIAL ELECTRONICS, VOL. 51, NO. 3, JUNE 2004.

[4] J. Elson, "Time synchronization in wireless sensor networks," Ph.D. dissertation, University of California, Los Angeles, May 2003.

[5] Ikuo Ihara Nagaoka University of Technology "Ultrasonic Sensing: Fundamentals and Its Applications to Non-destructive Evaluation (a draft)".

[6] ATMEGA32 Datasheet.

[7] A. Zelinsky1, R.A. Jarvis2, J.C. Byrne2 and S. Yuta3, "Planning Paths of Complete Coverage of an Unstructured Environment by a Mobile Robot", Intelligent Machine Behaviour Section, Electrotechical Laboratory, Tsukuba 305 Japan, in Proc. ICAR'93, 1993, pp. 533-538.

[8] Giuseppe Oriolo, Member, IEEE, Giovanni Ulivi, Member, IEEE, and MarilenaVendittelli , "Real-Time Map Building and Navigation for Autonomous Robots in Unknown Environments", IEEE TRANSACTIONS ON SYSTEMS, MAN, AND CYBERNETICS-PART B: CYBERNETICS, VOL. 28, NO. 3, JUNE 1998.

[9] David Flanagan, "Java In A Nutshell", 5th Edition.

[10] MIT Centre for Mobile Learning, Google's App Engine service, under the care of Massachusetts Institute of Technology (MIT) - USA.

[11] Patrick Reignier. Fuzzy logic techniques for mobile robotobstacle avoidance. Robotics and Autonomous Systems, 12:143 - 153, 1994. 